\title{
1 Dissection of intestines from larval zebrafish for molecular analysis
}

3 Bilge $\operatorname{San}^{1}$, Marco Aben $^{1,2,+}$, Gert Flik ${ }^{3}$, Leonie M. Kamminga ${ }^{1,2 *}$

$5{ }^{1}$ Radboud University Medical Center, Radboud Institute for Molecular Life Sciences, 274 Department

6 Molecular Biology, P.O. Box 9101, 6500 HB Nijmegen, The Netherlands, phone: +31(0)243616850, e-mail:

7 sanbilge@gmail.com, marco.aben@radboudumc.nl, I.kamminga@science.ru.nl, ${ }^{2}$ Radboud University,

8 Faculty of Science, Radboud Institute for Molecular Life Sciences, 274 Department Molecular Biology,

9 P.O. Box 9101, 6500 HB Nijmegen, The Netherlands, phone: +31(0)243616850, e-mail:

10 marco.aben@radboudumc.nl, I.kamminga@science.ru.nl, ${ }^{3}$ Radboud University, Faculty of Science,

11 Department of Animal Ecology and Physiology, P.O. Box 9010, 6500 GL Nijmegen, The Netherlands,

12 phone: +31(0)243653242, e-mail: g.flik@fnwi.ru.nl

$13{ }^{+}$Current affiliation: Radboud University Medical Center, Department of Human Genetics, P.O. Box 9101,

146500 HB Nijmegen, The Netherlands, marco.aben@radboudumc.nl

$15 *$ Corresponding author

16

\section{AUTHOR CONTRIBUTIONS}

18 B.S. conceived and designed the methodology and performed the experiments, analyzed the data, and

19 prepared figures. M.A. performed experiments. L.M.K. conceived and supervised the study, acquired

20 funding, was responsible for project management. B.S., G.F., and L.M.K. wrote the manuscript. All

21 authors reviewed the manuscript. 


\section{ABSTRACT}

24 Epigenetic data obtained from whole zebrafish embryos or larvae may mask or dilute organ-specific

25 information. Fluorescence activated cell sorting can diverge cells from their native state, and

26 cryosections often yield insufficient material for molecular analysis. Here, we present a reproducible

27 method for larval intestinal isolation at 5, 7, and 9 days post-fertilization, using the intestine-specific

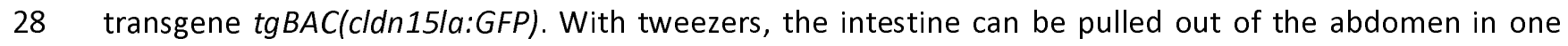

29 smooth motion. Upon removal of adhering tissues, intestines can be directly used for analyses. Each

30 dissection takes 3-6 minutes per fish. We demonstrate that 10 and 25 dissected intestines yield enough

31 material for RNA-sequencing and ChIP-sequencing, respectively. This method results in high quality, live

32 material, suitable for many downstream applications.

34 METHOD SUMMARY

35 We present a reproducible method for zebrafish larval intestinal isolation which results in high quality,

36 live material. With tweezers, the intestine can be pulled out of the abdomen and after removal of

37 adhering tissues, intestines can be directly used for analyses. We demonstrate that 10 and 25 dissected

38 intestines yield enough material for RNA-sequencing and ChIP-sequencing, respectively. 


\section{INTRODUCTION}

40 Genetic and epigenetic studies on zebrafish embryos and larvae require different, stage-dependent

41 approaches. Whole embryo lysates are commonly used for studies on gene expression and epigenetics

42 during early embryonic development [1-3]. However, as tissues and organs are specified, information

43 originating from a defined tissue may mask another and signals may 'dilute'. To eliminate noise and

44 increase reliability, isolation of specific tissues or cells of an organ becomes mandatory.

To obtain organ-specific information for whole genome (DNA) or transcriptome (RNA) analysis,

tissues can be dispersed and the cells sorted by fluorescence activated cell sorting (FACS) [4]. FACS enables the collection of specifically labeled living (single) cell populations out of a whole tissue or organism. It is a broadly applied method, for instance for blood cell subtyping [5]. However, cell surface markers might behave differently in single cell suspensions and might be cleaved by proteases (e.g.

Trypsin) [6]. In zebrafish, unlike mammals, there is limited availability for commercial antibodies for cell

51 surface markers, therefore, FACS is commonly used with transgenic lines which express tissue-specific

52 fluorescent proteins. Cells obtained by FACS can then be pooled for DNA (chromatin), RNA, protein extraction, or separated for single cell studies [7]. Long preparation times, however, decrease cellular yield [8] and lead to anoikis (i.e. apoptosis caused by absence of cellular contacts) [9]. Importantly, single cells from dissociated tissues may undergo transcriptional changes, including immediate early response gene activation (e.g. fos, jun, hsp gene variants) [10] and further alterations in cell signaling pathways

57 [11]. These alterations in dissociated cells can also cause dedifferentiation [12]. may also cause alterations from native cellular conditions. To assess gene expression in only a subset of

61 cells, cells can be extracted from tissue sections by carbon dioxide laser capture microdissection [14].

62 These methods are limited to RNA- and DNA-sequencing; a (part of a) single embryo or larva is currently 
63 insufficient for chromatin immunoprecipitation with commercially available antibodies, independent of

64 the stage of (early) development [15].

65 Dissection of organs is a common procedure in studies on adult zebrafish [16], while embryonic and larval dissection studies are uncommon or not well-described. The embryonic heart is the most commonly dissected organ due to its peripheral position in the body, and the broad research interest in its regenerative capacity [17-21]. The zebrafish pronephros, precursor of kidney tissue among others, is another organ which has been dissected at 5 days post-fertilization (dpf) to study gene expression by real-time quantitative PCR [22]. Zebrafish intestine is of great interest due to its rapid development, renewal potential, and its function in supplying nutrients to the larvae after yolk depletion. A number of laboratories have documented intestinal dissections, however, either the dissection method used has not been explained in detail, or the subsequent technical analysis did not require a pure intestine [2326]. Therefore, we investigated the feasibility of intestinal dissections in zebrafish larvae.

between 2-3 dpf [28]. During intestinal lumen formation, the liver and pancreas 'Anlagen' differentiate at proliferation [27]. Zebrafish is a stomachless species, and its intestine is clearly separated into three

81 parts: intestinal bulb, mid-intestine, and posterior intestine. By $5 \mathrm{dpf}$, the intestine becomes functional

82 with the opening of the mouth and anus, when most yolk is absorbed and the larva starts feeding

83 exogenously. To understand the regulatory processes in such a rapidly developing organ, the analysis of

84 different time-points becomes necessary. Throughout the second week of development, different

85 epithelial cell subtypes, namely enterocytes, goblet cells, enteroendocrine cells, and specialized antigen

86 presenting $(\mathrm{NaPi}+)$ enterocytes (in order of abundance) continue to differentiate $[29,30]$. With the 
87 growth of the intestine, this anterior loop folds into a sigmoid shape by adulthood [31]. Although the

88 zebrafish intestinal lining (an epithelium very rich in enterocytes) is very similar in structure to that of

89 mammals, it has ridges instead of villi. Proliferation, like in mammals in the crypts, predominantly occurs

90 at the base of these ridges [30].

91 We present a rapid and reproducible method of dissection of larval zebrafish intestine with the

92 aid of the intestine-specific transgenic line $\operatorname{tg} B A C(c l d n 15 / a: G F P)$, which expresses the GFP-tagged protein

93 'claudin 15-like a', an integral protein in the tight junctions of the intestinal epithelium [28]. We show

94 that this technique is compatible with methods such as RNA- and ChIP-sequencing, and surpasses the

95 efficiency of FACS of intestinal cells in the larval stages of this transgenic line. 
MATERIALS AND METHODS

97

Zebrafish strains and husbandry

99

Transgenic lines $\operatorname{tgBAC(cldn15/a:GFP)}$ [28] and $\operatorname{tg}(g u t: G F P)$ [32] were used for GFP expression in the intestine. Embryos were raised in E3 embryo medium at $28.5^{\circ} \mathrm{C}$ as described in detail elsewhere [33]. GFP expression was checked at 3-5 dpf under light anesthesia in 2-phenoxyethanol $(0.05 \% \mathrm{v} / \mathrm{v})$. Larvae were fed twice daily with dry feed (Gemma Micro 75, Skretting), rotifers, and artemia according to guidelines [33,34]. All experiments described are in accordance with institutional animal welfare guidelines, policies, and laws, and were approved after ethical testing by Central Committee for Animal Experimentation (CCD, approval number AVD1030020184668) of the Netherlands.

\section{Fluorescence Activated Cell Sorting}

108 Two hundred 5, 7 or $9 \mathrm{dpf}$ larvae in the $\operatorname{tg}($ gut:GFP) or $\operatorname{tgBAC}(\mathrm{cldn15}$ la:GFP) background were anesthetized, divided into $1.5 \mathrm{ml}$ microcentrifuge tubes (20 larvae per tube), and washed with PBS. The larvae were dissociated in $0.25 \% \mathrm{w} / \mathrm{v}$ Trypsin (Sigma), $1 \mathrm{mM}$ EDTA in PBS at $28.5^{\circ} \mathrm{C}$ for $50-70$ minutes.

111 After trypsinization was stopped with $1 \mathrm{mM} \mathrm{CaCl}_{2}$ and $100 \mu \mathrm{l} 100 \% \mathrm{FBS}$, the suspension was passed 112 through a FACS filter $(B D, 70 \mu \mathrm{m})$ and clarified with Dnase I $(100 \mu \mathrm{g} / \mathrm{ml})$. Finally, the suspension was 113 washed two times in PBS/1 mM EDTA solution and stained with 7-Aminoactinomycin D (7-AAD, Thermo 114 Fisher) for cell viability. GFP-positive cells were sorted by BD FACS-Aria into TRIzol (Thermo Fisher).

\section{Dissection}

117 A Petri dish lid was positioned under a fluorescence stereo microscope (Leica MZ FLIII) as a working 118 surface. During all steps, light microscopy and fluorescent microscopy were combined. Up to 4 $119 \operatorname{tg} B A C(c / d n 15 / a: G F P)$ larvae of 5, 7, or $9 \mathrm{dpf}$ were placed in 2-phenoxyethanol $(0.05 \% \mathrm{v} / \mathrm{v})$ for anesthesia 
and processed within 30 minutes following loss of startle response. One larva was transferred under the

121 microscope in 3-4 $\mathrm{ml}$ anesthesia medium, facing the dominant hand of the researcher. With one clean

122 watchmaker's tweezer, the larva was pierced rostrally to the intestine behind the branchial arches and

123 the intestine was clamped. At the same time, the fish was stabilized by pinching the swim bladder with

124 another watchmaker's tweezer (Figure $1 \mathrm{~A}$ and $1 \mathrm{~A}^{\prime}$ ). Next, in one movement, the intestinal tract was

125 carefully and slowly pulled out in the direction of the head; i.e. held by the distal segment and pulled

126 towards the head of the fish (Figure $1 \mathrm{~B}$ and $1 \mathrm{~B}^{\prime}$ ). The intestinal tract was bisected at the transition

127 between the esophagus and the intestine, and the carcass was discarded or lysed for genotyping (Figure

$1281 \mathrm{C}$ and $\left.1 \mathrm{C}^{\prime}\right)$. The intestine was cleaned up under the microscope in fresh medium (Figure $1 \mathrm{D}$ and $1 \mathrm{D}^{\prime}$ ). In

$1295 \mathrm{dpf}$ larvae, yolk remnants were removed. At all time points, the swim bladder was pinched off with

130 tweezers. Then, with tweezers and a microsurgical blade, liver and pancreas connections were cut off to

131 free the intestine. Remnants of muscles were peeled off with tweezers. The intestine was double-

132 checked for remaining adhering tissues (e.g. the autofluorescent gallbladder). The pure intestine was

133 washed in clean system water, and then transferred into a $1.5 \mathrm{ml}$ microcentrifuge tube with a Pasteur

134 pipette. The working surface was frequently refreshed. After dissection, a maximum of 15 intestines

135 were pooled on ice to prevent tissue damage before processing.

\section{RNA isolation}

138 Microcentrifuge tubes $(1.5 \mathrm{ml})$ were filled with 100 or $500 \mu \mathrm{l}$ TRIzol for 1 or 10 intestines, respectively.

139 Cells sorted by FACS were collected into $500 \mu \mathrm{l}$ TRIzol. Dissected intestines were transferred with a

140 Pasteur pipette onto the lid of the tubes with a minimum volume of system water, and promptly shaken

141 in TRIzol for lysis. RNA was isolated as described elsewhere [35]. After phase separation, in-column

142 DNase I treatment was performed (ZYMO Quick-RNA MicroPrep). Total RNA yield was measured by

143 fluorometric quantification (Qubit). 


\section{Chromatin immunoprecipitation}

145 To prevent adsorption of dissected intestines to the tubes, microcentrifuge tubes (1.5 $\mathrm{ml}$ ) were coated

146 with 5\% (w/v) BSA (Sigma) solution [36] for 15 minutes and dried. Thirty dissected intestines were cross-

147 linked in $1 \%$ paraformaldehyde (Electron Microscopy Sciences) for 15 minutes. The reaction was

148 quenched with $125 \mathrm{mM}$ glycine for 5 minutes, and washed 3 times with PBS. The intestines were lysed

149 (20 mM Tris-Cl pH 7.5, 70 mM KCl, 1 mM EDTA, 10\% glycerol, 0.125\% NP40, protease inhibitor cocktail

150 [Roche]) and sonicated (6 cycles of 30 seconds, Bioraptor ${ }^{\circledR}$ Pico) to extract $\sim 200$ bp chromatin fragments.

151 The chromatin was bound to protein A/G beads (Invitrogen, 1003D), incubated with anti-H3K4me3

152 (Millipore, $2 \mu \mathrm{g}$ ) or anti-H3K27me3 (Millipore, $2 \mu \mathrm{g}$ ) antibodies overnight, then eluted off the beads.

153 Input DNA concentration (1:6 fraction of total) and ChIP yield (5:6 fraction) was measured by

154 fluorometric quantification (Qubit). 


\section{RESULTS AND DISCUSSION}

156 In zebrafish, the intestine, which is the sole deliverer of energy (feed) to the animal, develops very

157 quickly and throughout early larval stages. The organization of the intestine at that moment is 158 comparable to the adult situation. Zebrafish intestinal anatomy is functionally comparable to the 159 anatomy of most higher vertebrates [27]. Zebrafish intestinal epithelium is organized in ridges with 160 somewhat larger dimensions compared to mammalian villus-crypts [30]. Proliferative regulation is 161 similar, e.g. with a crucial role for Wnt signaling, which appears conserved from zebrafish to mammals $162[37,38]$. Therefore, the zebrafish intestine is an attractive translational model to study human diseases 163 [39] and for fundamental research on (epi)genetic regulation [1]. For these reasons, we aimed to isolate 164 the intestine from larval stages. This study combines zebrafish developmental physiology with molecular biology and demonstrates a highly feasible technique to dissect the intestinal tract of zebrafish larvae in the $\operatorname{tgBAC(cldn15la:GFP)}$ transgenic background. It further presents yield of RNA extraction and chromatin immunoprecipitation from intestines, and shows that the total RNA yield from dissected intestine surpasses that of FACS-samples by at least 4-fold in our hands. Here we will discuss the rationale behind the development, advantages, and disadvantages of this method.

\section{Fluorescence Activated Cell Sorting (FACS)}

172 To study zebrafish intestinal (epi)genetics during the first days of larval development, we used two 173 previously described transgenic lines, $\operatorname{tg}(g u t: G F P)$ [32] and $\operatorname{tgBAC(cldn15/a:GFP)}$ [28]. The rationale was

174 that from these transgenes cell suspensions could be made, from which intestinal cells could be isolated 175 by FACS for further molecular analyses. The isolation of cells from both of these lines prior to FACS 176 analysis presented challenges. Preparation of isolated cell suspensions from whole larvae took over 2 177 hours, during which cell viability decreased to 60\%. GFP-positive and negative cells did not present a 178 distinct boundary to set reliable gates for sorting. The percentage of GFP-positive cells obtained from 5 
dpf larvae was as low as $0.1 \%$ for $\operatorname{tg}($ gut:GFP) (Figure $2 \mathrm{~A}$ ), and $1.9 \%$ for $\operatorname{tgBAC(cldn15/a:GFP)}$ (Figure 2B).

180 As the $\operatorname{tg}($ gut:GFP) line also expresses the construct in the liver and pancreas and gave such low FACS

181 yield, we decided the continue our research with the $\operatorname{tg} B A C(c l d n 15 / a: G F P)$ line.

Cell dissociation for FACS is a rigorous process for cells due to the stress of enzymatic digestion and trituration. Hard and soft tissues require different durations to dissociate, and the timing of 184 complete dissociation changes according to the age of embryos/larvae. During cell dissociation, some cell death occurs and the viscous texture of genomic DNA in solution may encumber pipetting. As mentioned before, changes might occur in transcription, cell signaling pathways, and the differentiation 187 status of the cells [10-12].

\section{Intestinal dissection}

Next, we investigated whether segmenting the larvae into smaller parts prior to single cell preparations would increase the FACS yield and reduce noise from auto-fluorescence. For this inquiry, we attempted dissections on larval intestines in the $\operatorname{tgBAC(cldn15la:GFP)}$ background. Remarkably, these dissections were very consistent and time efficient. Moreover, the reduction of material processed per zebrafish greatly reduced the trypsinization time as well; approximately by half. Nonetheless, the FACS yield of this semi-pure population of GFP-positive cells was only $8.9 \%$ at $9 \mathrm{dpf}$ (Figure 2C, Supplementary Figure 1). Due to this (unexpected) low yield, we concluded that $\operatorname{tgBAC(cldn15/a:GFP)}$ is an unsuitable model for

FACS. However, it could serve as a great tool for obtaining intestine-specific cells through dissections. 
under the microscope. Moreover, the carcass from each larva can be genotyped, and any single unsuitable sample can be discarded before intestinal pooling. prevent the adherence of intestines to plastic, glass Pasteur pipettes were used for transfer of intestines, and BSA coating was chosen over the rather costly and inefficient use of dichlorodimethylsilane coating or low binding microentrifuge tubes. Bovine serum albumin (BSA) is a protein commonly used for

218 blocking Western blot membranes and ChIP beads, but also for coating laboratory equipment against adherence of materials [40].

Intestinal dissections can be considered as a difficult process prone to errors and variation.

221 Between 5-9 dpf, the length of the larvae is between 3.9-4.5 mm, which requires the use of a microscope

222 and watchmaker's tweezers. For the tissue to stay intact and unchanged from the start of the dissection

223 to tissue lysis/fixation, the researcher needs to act fast and gentle at the same time. Individual variation

224 in physiology [41] also needs to be considered for analysis of multiple fish. 
between the intestinal bulb and mid-intestine during the removal of the intestine from the body. The muscles are the most challenging extra-intestinal tissue to remove at $5 \mathrm{dpf}$ due to the fragility of the intestine and the thinness of the muscle lining. By $7 \mathrm{dpf}$, the intestine has hardened enough such that muscle, liver, and pancreas can be swiftly peeled/cut off of the intestine with the help of tweezers and microsurgical blades.

Further, the procedure is potentially prone to contamination with liver, pancreas, muscle, and

233 gallbladder. At $5 \mathrm{dpf}$, yolk contamination is also an additional risk. Therefore, at least 6 biological 234 replicates should be used if the extracted RNA will be used for sequencing [42]. For ChIP-sequencing, 235 more than two replicates are recommended to minimize errors in bioinformatics analysis [43]. Another 236 aspect of sample variation is the presence of four different epithelial cell types and three different 237 morphological segments within the intestine [29,30], with different functional properties [31]. It is a 238 coherent presumption that these different functions start developing before or during larval stages.

\section{RNA isolation and chromatin immunoprecipitation}

241 We used dissected intestines for RNA isolation and chromatin immunoprecipitation. At all time points, 242 total RNA obtained by single intestines (average: $28.4 \mathrm{ng}$ ) and pools of 10 intestines (average: $343 \mathrm{ng}$ )

243 was proportional to the number of larvae (Table 1). Surprisingly, FACS on intestinal cells at 9 dpf from 20

244 larvae yielded a proportionally >4-fold lower amount of total RNA than dissected intestines (Table 1).

245 The amount of RNA from dissected pooled intestines suffices as input for RNA-sequencing (Ribo-Zero 246 rRNA Removal Kit, Illumina) for all developmental time points used. To immunoprecipitate intestinal chromatin, we collected intestines in a BSA-coated 248 microcentrifuge tube. Immunoprecipitation of chromatin from 25 pooled intestines with anti-H3K4me3 249 and anti-H3K27me3 antibodies yielded sufficient starting material for Illumina sequencing preparation; 250 on average $6.3 \mathrm{ng}$ and $12.8 \mathrm{ng}$ chromatin was immunoprecipitated with anti-H3K4me3 and anti- 
251 H3K27me3, respectively (Table 2). We have used total RNA and chromatin from pooled zebrafish larval

252 intestines to analyze the wild type intestinal transcriptome and the presence of H3K4me3 and

253 H3K27me3 chromatin marks on gene promoters at 5, 7, and $9 \mathrm{dpf}$ [44]. To detect individual variation,

254 single intestines should be processed with available low input protocols [45-47]. However, low-input

255 ChIP yields still predominantly depend on the antibody efficiency, and the methods are costly for many

256 [48].

257 Although dissected intestines are a far better model than whole larvae for molecular and

258 biochemical analysis of the intestine, we recommend additional validation experiments such as staining

259 of individual mRNA or proteins to assess their localization. Additionally, dissected intestines can be used

260 for protein isolation and subsequent proteomics. In summary, intestinal dissection serves as an excellent

261 tool to compare differences in this rapidly developing organ between larval stages, and between wild

262 types and mutants. 


\section{ACKNOWLEDGEMENTS}

264 The authors would like to thank Tom Spanings and Antoon van der Horst of Radboud University for

265 zebrafish husbandry, Rob Woestenenk of the Radboud University Medical Center for FACS assistance,

266 Cornelia Veelken of the Radboud Institute for Molecular Life Sciences for sharing the FACS protocol,

267 Karolina Andralojc of the Radboud University for discussions on BSA-coating, Silvia Boj of the Hubrecht

268 Institute for the $\operatorname{tg}$ (gut:GFP) line, Ashley Alvers Lento and Michel Bagnat of Duke University for providing

269 the $\operatorname{tg} B A C(c l d n 15 / a: G F P)$ line.

270 
272 1. Lindeman LC, Andersen IS, Reiner AH, et al. Prepatterning of developmental gene expression by

273 modified histones before zygotic genome activation. Dev Cell. 21(6):993-1004 (2004).

274 2. Vastenhouw NL, Zhang Y, Woods IG, et al. Chromatin signature of embryonic pluripotency is 275 established during genome activation. Nature. 464(7290):922-6 (2010).

276 3. Kaaij LJT, Mokry M, Zhou M, et al. Enhancers reside in a unique epigenetic environment during early 277 zebrafish development. Genome Biology. 17(1):146 (2016).

278 4. Sugiyama, T., Kim, S.K. Fluorescence-activated cell sorting purification of pancreatic progenitor cells. 279 Diabetes Obes Metab. 10(Suppl 4):179-85 (2008).

280 5. Herzenberg LA, Parks D, Sahaf B, Perez O, Roederer M, Herzenberg LA. The History and Future of the 281 Fluorescence Activated Cell Sorter and Flow Cytometry: A View from Stanford. Clinical Chemistry, $28248(10): 1819-1827$ (2002).

283 6. Besingi RN, Clark PL. Extracellular Protease Digestion to Evaluate Membrane Protein Cell Surface 284 Localization. Nature Protocols. 10(12):2074-2080 (2015).

285 7. Grün, D., van Oudenaarden, A. Design and Analysis of Single-Cell Sequencing Experiments. Cell. 286 163(4):799-810 (2015).

287 8. Chang JP, Jobin RM: Teleost pituitary cells: isolation, culture and use. Analytical techniques (pp. 207). 288 Elsevier, Amsterdam, The Netherlands (1994).

289 9. Zhao B, Li L, Wang L, et al. Cell detachment activates the Hippo pathway via cytoskeleton 290 reorganization to induce anoikis. Genes \& Development, 26(1):54-68 (2012). 
291 10. van den Brink SC, Sage F, Vértesy Á, et al. Single-cell sequencing reveals dissociation-induced gene 292 expression in tissue subpopulations. Nat Methods. 14(10):935-936 (2017).

293 11. Geller SF, Lewis GP, Fisher SK. FGFR1, signaling, and AP-1 expression after retinal detachment: 294 reactive Müller and RPE cells. Invest Ophthalmol Vis Sci. 42(6):1363-9 (2001).

295 12. Minami K, Okano H, Okumachi A, Seino S. Role of cadherin-mediated cell-cell adhesion in pancreatic 296 exocrine-to-endocrine transdifferentiation. J Biol Chem. 283(20):13753-61 (2008).

297 13. Kruse F, Junker JP, van Oudenaarden A, Bakkers J. Tomo-seq: A method to obtain genome-wide 298 expression data with spatial resolution, Methods in Cell Biology, 135:299-307 (2016).

299 14. Emmert-Buck MR, Bonner RF, Smith PD, et al. Laser capture microdissection. Science. 274(5289):998$3001001(1996)$

15. Crosetto N, Bienko M, van Oudenaarden A. Spatially resolved transcriptomics and beyond. Nat Rev 302 Genet. 16(1):57-66 (2015).

16. Gupta T, Mullins MC. Dissection of Organs from the Adult Zebrafish. JoVE, 37(3):1717 (2010).

17. Kikuchi K. Advances in understanding the mechanism of zebrafish heart regeneration. Stem Cell Res.

305 13(3 Pt B):542-55 (2014).

18. Noël ES, Verhoeven M, Lagendijk AK, et al. A Nodal-independent and tissue-intrinsic mechanism controls heart-looping chirality. Nat Commun. 4:2754 (2013). (2006). 
310 20. Singleman C, Holtzman NG. Heart Dissection in Larval, Juvenile and Adult Zebrafish, Danio rerio. JoVE, $31155(9): 3165(2011)$.

312 21. Lombardo VA, Otten C, Abdelilah-Seyfried S. Large-scale Zebrafish Embryonic Heart Dissection for 313 Transcriptional Analysis. JoVE, 95(1):52087 (2015).

314 22. Corre T, Arjona FJ, Hayward C et al. Genome-Wide Meta-Analysis Unravels Interactions between 315 Magnesium Homeostasis and Metabolic Phenotypes. J Am Soc Nephrol. 29(1):335-348 (2017).

316 23. Enya S, Kawakami K, Suzuki Y, Kawaoka S. A novel zebrafish intestinal tumor model reveals a role for 317 cyp7a1-dependent tumor-liver crosstalk in causing adverse effects on the host. Disease models \& 318 mechanisms, 11(8),pii:dmm032383 (2018).

319 24. Rolig AS, Parthasarathy R, Burns AR, Bohannan BJ, Guillemin K. Individual Members of the Microbiota 320 Disproportionately Modulate Host Innate Immune Responses. Cell host \& microbe, 18(5):613-20 (2015).

321 25. Clifton JD, Lucumi E, Myers MC, et al.. Identification of novel inhibitors of dietary lipid absorption 322 using zebrafish. PloS one, 5(8):e12386 (2010).

323 26. Simonson LW, Ganz J, Melancon E, Eisen JS. Characterization of enteric neurons in wild-type and 324 mutant zebrafish using semi-automated cell counting and co-expression analysis. Zebrafish, 10(2):147-53 325 (2013).

326 27. Wallace KN, Pack M. Unique and conserved aspects of gut development in zebrafish. Dev Biol. 327 255(1):12-29 (2003).

328 28. Alvers AL, Ryan S, Scherz PJ, Huisken J, Bagnat M. Single continuous lumen formation in the zebrafish 329 gut is mediated by smoothened-dependent tissue remodeling. Development, 141(5):1110-1119 (2014). 
331 Intestinal epithelium morphogenesis, Developmental Biology, 286(1):114-135 (2005).

332 30. Wallace KN, Akhter S, Smith EM, Lorent K, Pack M. Intestinal growth and differentiation in zebrafish.

333 Mechanisms of Development, 122(2):157-173 (2005).

334 31. Wang Z, Du J, Lam SH, Mathavan S, Matsudaira P, Gong Z. Morphological and molecular evidence for 335 functional organization along the rostrocaudal axis of the adult zebrafish intestine. BMC Genomics, $33611: 392$ (2010).

337 32. Field HA, Ober EA, Roeser T, Stainier DY. Formation of the digestive system in zebrafish. I. liver 338 morphogenesis, Developmental Biology, 253(2):279-290 (2003).

33. Westerfield, M: The zebrafish book, A guide for the laboratory use of zebrafish (Danio rerio), 5th ed., 340 University of Oregon Press, Eugene, Oregon, the United States of America (2007).

34. Wilson C. Aspects of larval rearing. ILAR J. 53(2):169-78 (2012).

35. Chomczynski P. A reagent for the single-step simultaneous isolation of RNA, DNA and proteins from

343 cell and tissue samples. BioTechniques, 15(3):532-537 (1993).

344 36. Bratcher PE, Gaggar A. Characterization and Prevention of the Adsorption of Surfactant Protein D to 345 Polypropylene. PLOS ONE, 8(9):e73467 (2013).

346 37. Cheesman SE, Neal JT, Mittge E, Seredick BM, Guillemin K. Epithelial cell proliferation in the 347 developing zebrafish intestine is regulated by the Wnt pathway and microbial signaling via Myd88. PNAS. 348 108(Suppl 1):4570-4577 (2011). 
39. Lu J-W, Ho Y-J, Ciou S-C, Gong Z. Innovative Disease Model: Zebrafish as an In Vivo Platform for

352 Intestinal Disorder and Tumors. Biomedicines, 5(4):58 (2017).

353 40. Man Y, Lv X, Iqbal J et al. Adsorptive BSA Coating Method for CE to Separate Basic Proteins

354 Chromatographia. 76(1-2):59-65 (2013).

355 41. Field HA, Kelley KA, Martell L, Goldstein AM, Serluca FC. Analysis of gastrointestinal physiology using

356 a novel intestinal transit assay in zebrafish. Neurogastroenterology and Motility. 21(3):304-12 (2009).

42. Schurch NJ, Schofield P, Gierliński M et al. How many biological replicates are needed in an RNA-seq experiment and which differential expression tool should you use? RNA, 22(6):839-851 (2016). Computational and Structural Biotechnology Journal, 9:e201401002 (2014).

44. 48. San B, Aben M, Elurbe DM et al. Genetic and epigenetic regulation of zebrafish intestinal development. Epigenomes. 2(4):19 (2018).

45. Dahl JA, Gilfillan GD. How low can you go? Pushing the limits of low-input ChIP-seq. Briefings in Functional Genomics, 17(2):89-95 (2018).

46. Picelli S, Faridani OR, Björklund AK, Winberg G, Sagasser S, Sandberg R. Full-length RNA-seq from single cells using Smart-seq2. Nature Protocols. 9(1):171-81 (2014). amplification. Cell Reports. 2(3):666-73 (2012). 


\section{FIGURE LEGENDS}

373 Figure 1. FACS sorting in $\operatorname{tg}($ gut:GFP) and $\operatorname{tg}(\mathrm{cldn15la:GFP).} \mathrm{Single} \mathrm{cell} \mathrm{suspensions} \mathrm{were} \mathrm{prepared} \mathrm{from}$

$3745 \mathrm{dpf}$ whole zebrafish larvae in the $\operatorname{tg}(g u t: G F P)(\mathbf{A})$ and $\operatorname{tg}(\mathrm{cldn15}$ la:GFP) (B) backgrounds. The GFP-

375 positive populations (A, B, purple dots) were gated according to the GFP-negative population (A, B, black

376 dots) and sorted into TRIzol for RNA extraction. C. FACS on whole $\operatorname{tg}(g u t: G F P)$ and $\operatorname{tg}(c l d n 15 / a: G F P)$ larval

377 suspensions at $5 \mathrm{dpf}$ yielded $0.1 \%$ and $1.9 \%$ GFP-positive cells, respectively. Unexpectedly, FACS on

378 dissected intestines from $\operatorname{tg}(\mathrm{cldn15la:GFP)}$ yielded as low as 8.9\% GFP-positive cells.

Figure 2. Dissection of the larval intestine. Overview of the major steps during the dissection of a larval intestine; $5 \mathrm{dpf}$ is shown as an example. The same steps are taken for 7 and $9 \mathrm{dpf}$. Left and right panels are the same field of view under light microscopy $(A, B, C, D)$ and fluorescent microscopy $\left(A^{\prime}, B^{\prime}, C^{\prime}, D^{\prime}\right)$, respectively. A, A'. With the help of tweezers, the intestine was stabilized. B, $\mathbf{B}^{\prime}$. The intestine was slid out of the body in the direction of the head. C, C'. The extra-intestinal tissues were cleaned off by peeling or cropping by microsurgical blades. D, D'. The intestine was carefully checked for GFP purity and promptly transferred into a microcentrifuge tube.

Table 1. Total RNA yield. Total RNA was isolated from single or a pool of 10 dissected intestines at 5, 7, and $9 \mathrm{dpf}$ in triplicates, and the yield was quantified fluorometrically (Qubit). The average yield is shown in nanograms. FACS-sorted intestinal cells from 20 whole $\operatorname{tg}(\mathrm{cldn15/a:GFP)} \mathrm{larvae} \mathrm{at} 10 \mathrm{dpf}$ yielded 4-fold less total RNA.

Table 2. Chromatin immunoprecipitation yield. After chromatin extraction from 30 intestines at 5, 7, or

$3949 \mathrm{dpf}$, samples were sonicated to obtain 200 bp fragments, and one sixth of the DNA ( 5 intestines) was 
396 H3K4me3 or anti-H3K27me3 immunoprecipitation in replicates, eluted off, and the yield was quantified

397 fluorometrically (Qubit). The average ChIP yield is shown in nanograms.

398

399 Supplemental Figure 1. FACS sorting in dissected tg(cldn15la:GFP) intestines. Intestines of $10 \mathrm{dpf}$ were

400 crudely dissected from $\operatorname{tg}(\mathrm{cldn15la:GFP)}$ larvae and single cell suspensions were prepared. GFP-negative

401 population (left panel, red dots) was used to gate the GFP-positive population (right panel, red dots).

402 GFP-positive population was calculated as $8.9 \%$.

403 

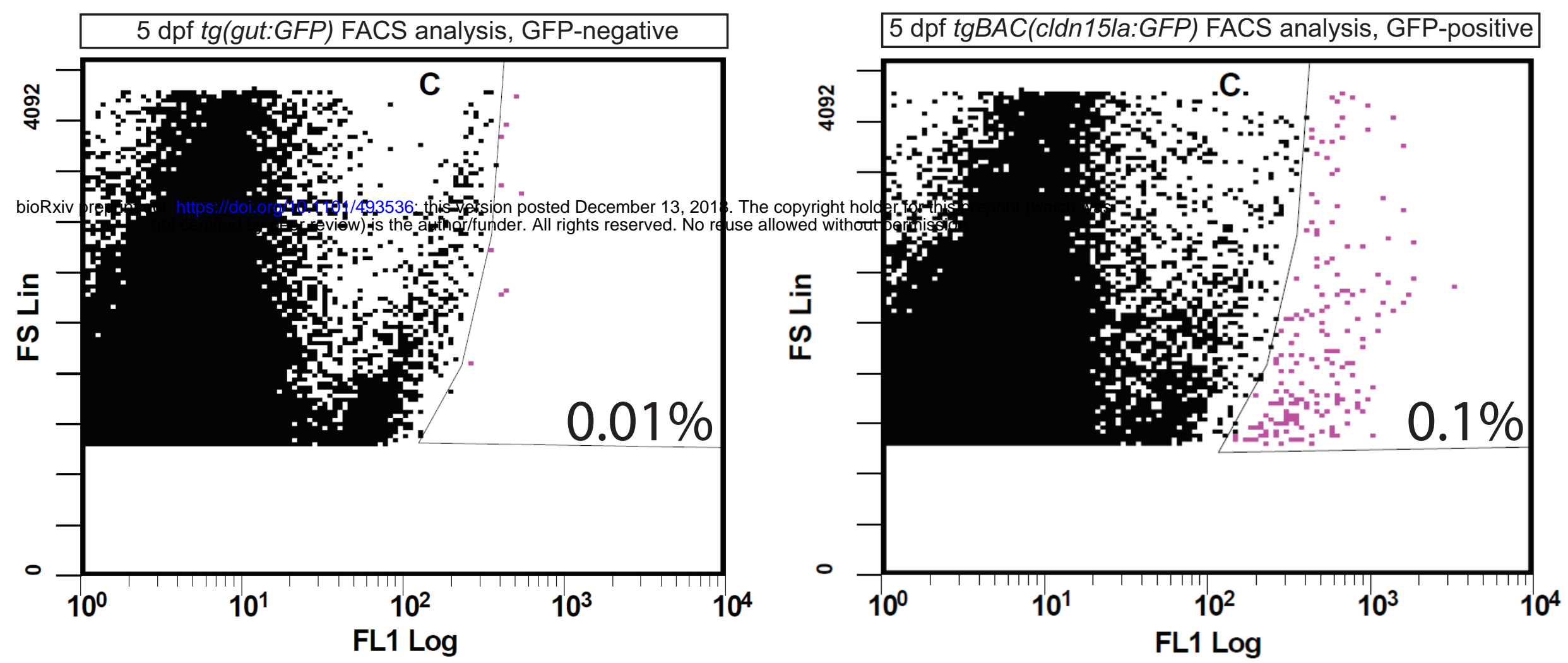

B
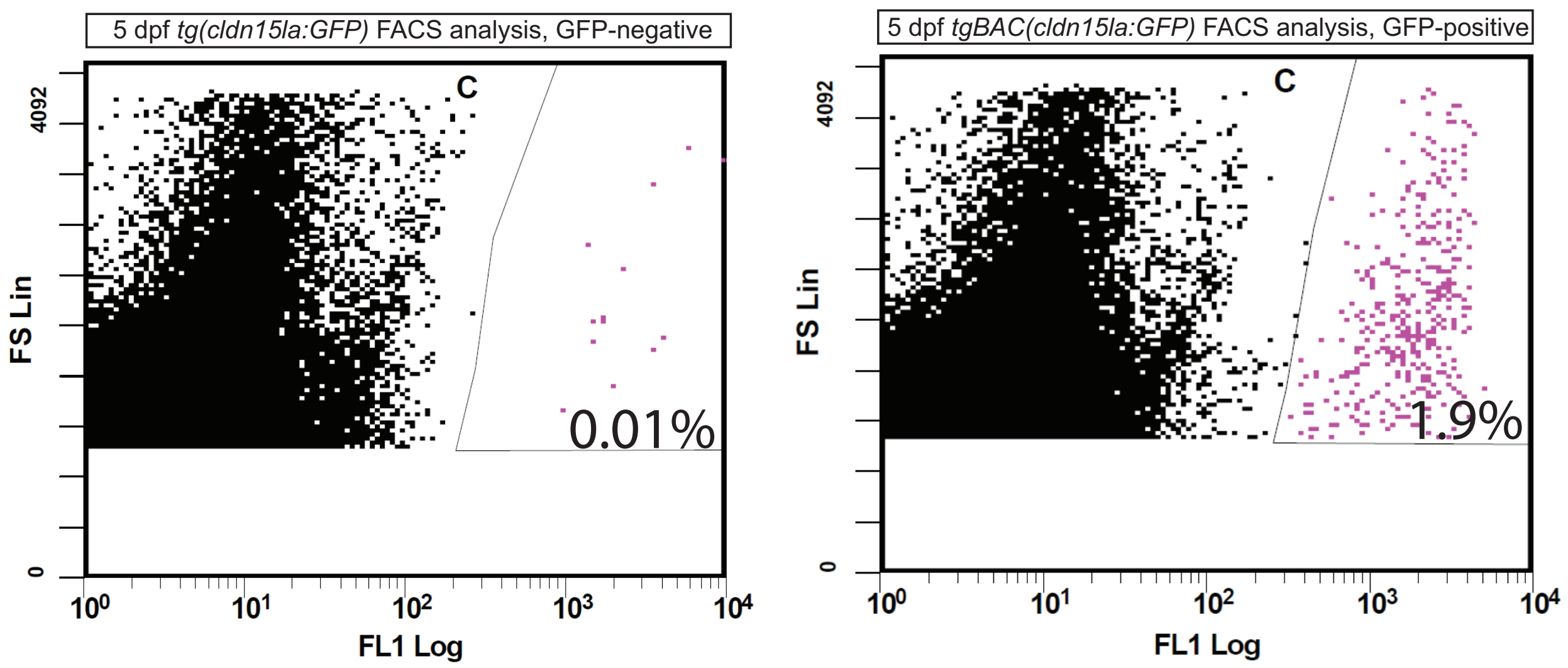

C

\begin{tabular}{|c|c|}
\hline & GFP+ percentage \\
\hline $\operatorname{tg}($ gut:GFP), whole & $0.1 \%$ \\
\hline $\operatorname{tg} B A C($ cldn15la:GFP), whole & $1.9 \%$ \\
\hline $\operatorname{tgBAC}($ cldn15la:GFP), dissected & $8.9 \%$ \\
\hline
\end{tabular}




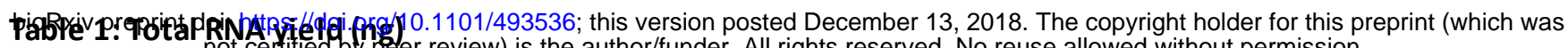

\begin{tabular}{|l|c|c|c|}
\hline \multirow{2}{*}{ Single intestines } & 24.4 & 30.8 & 29.9 \\
\hline 10 intestines & 364.5 & 348 & 316.5 \\
\hline FACS (20 larvae) & N/A & N/A & 150 \\
\hline
\end{tabular}




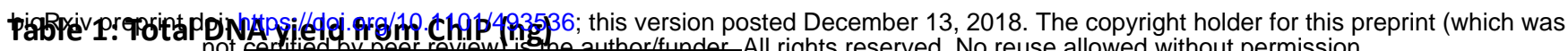

\begin{tabular}{|l|c|c|c|}
\hline \multicolumn{1}{c}{} & $5 \mathrm{dpf}$ & $7 \mathrm{dpf}$ & $9 \mathrm{dpf}$ \\
\hline anti-H3K4me3 & 8.4 & 6.4 & 4.2 \\
\hline anti-H3K27me3 & 14.2 & 11.8 & 12.4 \\
\hline Input & 56.4 & 76.4 & 141.2 \\
\hline
\end{tabular}

